EPJ Web of Conferences 24, 02002 (2012)

DOI: $10.1051 /$ epjconf/20122402002

(C) Owned by the authors, published by EDP Sciences - SIF, 2012

\title{
Radioactivity measurements for the ERMES project at the STELLA facility
}

\author{
M. Laubenstein ${ }^{1}$, W. Plastino ${ }^{2,3}$, P. Aprili $^{1}$, M. Balata $^{1}$,

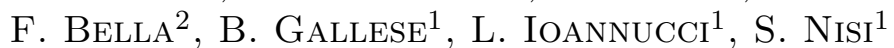

${ }^{1}$ INFN, Gran Sasso National Laboratory - I-67100 Assergi, Italy

2 Department of Physics, University of Roma Tre - I-00146 Rome, Italy

${ }^{3}$ INFN, Section of Roma Tre - I-00146 Rome, Italy

\begin{abstract}
STELLA (SubTErranean Low Level Assay) is the ultra low background facility of the Gran Sasso National Laboratories (L.N.G.S.) in Italy. It is mainly devoted to material screening and rare events physics due to its very low radioactive background. Nevertheless, also environmental samples are measured within the collaboration with the ERMES (Environmental Radioactivity Monitoring for Earth Sciences) project. After a short description of the facility some on-going applications within the ERMES project will be briefly presented. The usefulness of doing environmental radioactivity measurements in a deep underground laboratory will be shortly discussed.
\end{abstract}

\section{Introduction}

The Gran Sasso National Laboratories in the centre of Italy host the world's biggest underground laboratories [2]. The three big experimental halls are shielded against the cosmic radiation by $1400 \mathrm{~m}$ of rock overburden, beneath the Gran Sasso Massif. This rock overburden, corresponding to approximately 3800 metres of water equivalent, reduces the muon flux one million times with respect to the Earth's surface. Moreover, it diminishes the neutron background by a factor of one thousand, leaving mainly the component of neutrons produced by nuclear reactions due to natural radioactivity

This is an Open Access article distributed under the terms of the Creative Commons Attribution License 2.0, which permits unrestricted use, distribution, and reproduction in any medium, provided the original work is properly cited. 
(uranium and thorium decay chains). Occasionally, the intrinsic radioactive concentration of the surrounding rock is rather low, as it is mainly of dolomitic nature, which minimizes the gamma ray background significantly with respect to other underground laboratories, surrounded by granitic rock. The L.N.G.S. are housing large and medium scale experiments focused on Astroparticle and Neutrino Physics in the three experimental halls. They have also a service facility [1], which was recently given the name STELLA, devoted in first priority to the following items:

- material screening for experiments installed at the L.N.G.S.;

- small fundamental physics research projects;

- interdisciplinary research projects.

The main measurement technique used is gamma ray spectroscopy with 10 high purity Germanium (HPGe) detectors. In order to be as sensitive as possible for the measurement of natural radioactivity (uranium and thorium decay chains, potassium) all detectors have been built in so-called ultra low background (ULB) configuration. This term means that all materials used for the detector have been carefully selected for their intrinsic content of the primordial nuclides. More details on their configuration can be found in [1].

\section{Environmental measurements}

It could be questioned whether it is really necessary to perform environmental measurements with uranium and thorium concentrations at the level of $10^{-6} \mathrm{~g} \mathrm{~g}^{-1}$ in conditions of extremely low background as in an underground laboratory. For sure, the measurement of an environmental sample can be assumed as background free, because the intrinsic background count rate of the ULB detector system is 1000 times lower than the one of the sample. In addition the variation of the background rate is negligible as the contributions from cosmic rays and radon in the air are almost completely absent. This fact eliminates one rather important contribution to the systematic uncertainty, which is always difficult to estimate and rather cumbersome to deal with. Moreover, within the limits of the experimental procedures, the measurement time can be reduced rather significantly, which allows for a higher throughput in case of a large number of samples to be screened. Overall the result of the measurement is more robust, due to the very low and rather constant background.

In the following two ongoing projects of the ERMES experiment will be briefly described. 


\subsection{The Solforata lake}

Close to Rome, near Pomezia exists one of the many areas with volcanic activity in this region, the Solforata lake. In order to study the correlation between in-situ measurements performed with a handheld high purity Germanium (HPGe) detector (ORTEC Detective-EX-100) and laboratory measurements with HPGe detectors a measurement campaign was organised. Within this campaign 66 soil/mud samples were taken following a regular grid in four different areas around the lake for the laboratory measurement. At the sampling sites also in-situ measurements were performed. The laboratory samples were all measured at the L.N.G.S. by means of gamma ray spectroscopy. In table I are reported the average values, and the minimum and maximum values of each area for the naturally occurring radioisotopes obtained by the laboratory measurements. As can be seen in the table the values of ${ }^{228} \mathrm{Ra}$ and ${ }^{228} \mathrm{Th}$ clearly show the presence of secular equilibrium within the ${ }^{232} \mathrm{Th}$ decay chain. The same holds for the ${ }^{238} \mathrm{U}$ chain looking at ${ }^{226} \mathrm{Ra}$ and ${ }^{238} \mathrm{U}$ (via ${ }^{234 m} \mathrm{~Pa}$ ). The variability within the grid is quite high, which indicates very localized variations in the radionuclide concentrations. The comparison between laboratory measurements and in-situ measurements have been published recently in [3]. They show a good agreement between the two methods. The work showed also that a comparison between the results for ${ }^{226} \mathrm{Ra}$ obtained in-situ and in the laboratory can be a good probe to identify and locate the presence of exhalation of ${ }^{222} \mathrm{Rn}$. The work is currently going on in order to estimate the amount of ${ }^{222} \mathrm{Rn}$ emitted in the area.

\subsection{The regione Molise}

Italy is divided into administrative areas called regioni. One of the smallest ones is Molise in the Central part of Italy. The civil protection services of Molise decided to create a map concerning the natural radioactivity of the whole regione in order to comply with the national law prescriptions. These prescriptions foresee such a mapping in order to better assess possible implications due to any scenario which might involve accidental contamination of the territory with radioactive material. Within the ERMES project 206 soil samples were collected in uncultivated ground by spade following a regular grid layed over the whole area of Molise. They were measured in the STELLA facility of the L.N.G.S. The samples were not treated at all, which is not the standard procedure, where the samples are dried and then homogenized. The Monte Carlo simulation programs used for the cal- 
Table I: Average and minimum/maximum concentrations of the radionuclides ${ }^{238} \mathrm{U}$, ${ }^{226} \mathrm{Ra},{ }^{228} \mathrm{Ra},{ }^{228} \mathrm{Th}$ and ${ }^{40} \mathrm{~K}$ for the four areas at the Solforata Lake under investigation, obtained via gamma ray spectroscopy at the L.N.G.S.

\begin{tabular}{rrcccc}
\hline Radionuclide & & Area 1 & Area 2 & Area 3 & Area 4 \\
\hline \hline${ }^{238} \mathrm{U}$ & average & $126 \pm 48$ & $182 \pm 65$ & $93 \pm 44$ & $132 \pm 41$ \\
& minimum & 51 & 103 & 34 & 87 \\
& maximum & 248 & 339 & 201 & 223 \\
\hline${ }^{226} \mathrm{Ra}$ & average & $176 \pm 57$ & $138 \pm 40$ & $109 \pm 53$ & $82 \pm 22$ \\
& minimum & 107 & 77 & 43 & 54 \\
& maximum & 325 & 214 & 276 & 124 \\
\hline${ }^{228} \mathrm{Ra}$ & average & $224 \pm 42$ & $262 \pm 31$ & $187 \pm 93$ & $227 \pm 37$ \\
& minimum & 174 & 226 & 35 & 176 \\
& maximum & 322 & 335 & 340 & 294 \\
\hline${ }^{228} \mathrm{Th}$ & average & $220 \pm 43$ & $250 \pm 29$ & $181 \pm 90$ & $217 \pm 35$ \\
& minimum & 172 & 213 & 32 & 172 \\
& maximum & 329 & 321 & 327 & 275 \\
\hline${ }^{40} \mathrm{~K}$ & average & $269 \pm 90$ & $346 \pm 117$ & $257 \pm 122$ & $285 \pm 89$ \\
& minimum & 111 & 199 & 74 & 158 \\
& maximum & 400 & 577 & 601 & 434 \\
\hline
\end{tabular}

culation of the counting efficiency are able to take into account the different conditions of each sample (inhomgeneities, density). After the measurement the samples were dried and the results normalized to the dry weight. The dose rates due to the naturally occurring radionuclides $\left({ }^{238} \mathrm{U},{ }^{232} \mathrm{Th}\right.$ and ${ }^{40} \mathrm{~K}$ ) were calculated according to the formalism given in the UNSCEAR 2000 report [4]. As the altitude of each sample was well documented we calculated also the dose rate due to cosmic rays at the sampling site, again according to the formalism given in the UNSCEAR 2000 report. Although in some samples also ${ }^{137} \mathrm{Cs}$ was found, which is due to fall-out from atmospheric bomb tests and nuclear incidents, we did not take it into account, as our sampling method was not designed for a quantitative determination of this radionuclide. Anyhow, a rough estimate gives only a very small contribution of ${ }^{137} \mathrm{Cs}$ to the total dose rate (less than 5\%). A map of the total dose rate (from gamma radiation of naturally occurring radioisotopes and cosmic rays, but excluding ${ }^{137} \mathrm{Cs}$ ) was created, which is shown in figure 1 . As can be seen in the figure the dose rate is rather homogeneously distributed over the whole region. The average value is $101 \mathrm{nGy} \mathrm{h} \mathrm{h}^{-1}\left(65 \mathrm{nGy} \mathrm{h} \mathrm{h}^{-1}\right.$ from naturally occurring radioisotopes, $36 \mathrm{nGy} \mathrm{h}^{-1}$ from cosmic rays). There are 


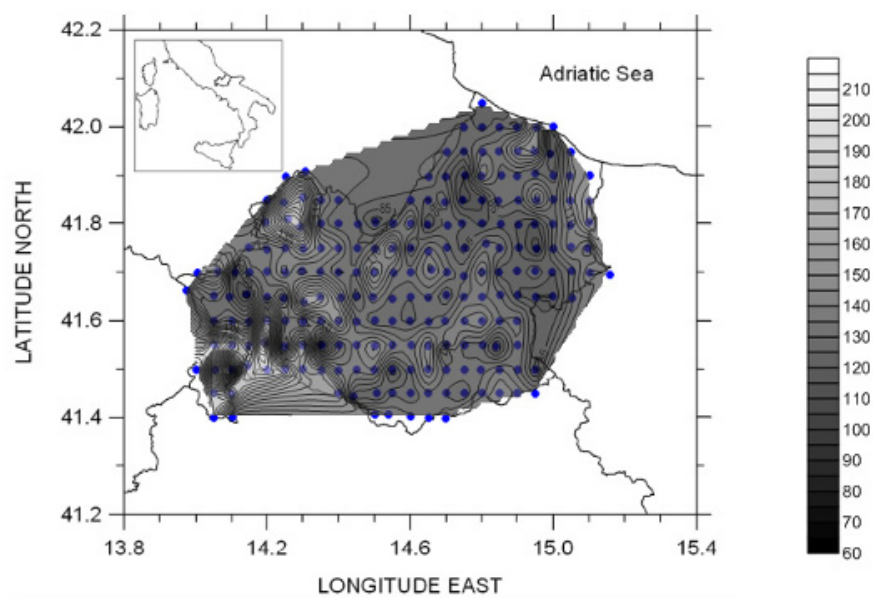

Figure 1: Map of dose rates in the regione Molise including the part deriving from cosmic radiation and the one from natural radioactivity in the soil. The numbers at the greyscale code on the right are given in $\mathrm{nGy} \mathrm{h}^{-1}$.

some hot spots (maximum value $215 \mathrm{nGy} \mathrm{h}^{-1}$ ) that are mostly related to the areas where the concentration of thorium and radium is higher (southeast of the Molise). The average value of the dose rate from natural radioactivity is less than the average value of $72 \mathrm{nGy} \mathrm{h}^{-1}$ for all Italy. The correlation between the concentration of the naturally occurring radionuclides and the orography of the Molise is currently under investigation.

The authors express their gratitude to the Director of the L.N.G.S. and to the L.N.G.S. staff for the effective and continuous support.

\section{References}

[1] Arpesella C., Appl. Radiat. Isot., 47 (1996) 991.

[2] Coccia E., Nucl. Phys. Proc. Suppl., 139 (2005) 3. 
[3] Di Paolo F. et al., 2011 Seventh Conference on Natural Computation, doi 10.1109/ICNC.2011.6022594 (2011) p. 2100-2103.

[4] UNSCEAR, Report of the United Nations Scientific Committee on the Effects of Atomic Radiation to the General Assembly, with Scientific Annexes (United Nations, New York) 2000. 